\title{
Integer Solutions of Non-Homogeneous Ternary Cubic Equation $x^{2}+y^{2}-x y=103 z^{2}$
}

\author{
Dr. P. Jayakumar ${ }^{1}$, J. Meena ${ }^{2}$ \\ ${ }^{1}$ Professor of Mathematics, Periyar Maniammai University, Vallam, Thanajvur -613 403, Tamil Nadu, India \\ ${ }^{2}$ Ph.D. Scholar, Assistant Professor of Mathematics, A.V.V.M. Sri Pushpam College Poondi -613 503, Thanajvur
}

\begin{abstract}
The non- homogeneous ternary cubic equation given by $x^{2}+y^{2}-x y=103 z^{2}$ is analyzed for its non-zero distinct integer solutions. Introducing the linear transformations $x=u+v, y=u-v(u \neq v \neq 0)$ and applying the methods of factorization, different patterns of integer solutions of the above equations are obtained.
\end{abstract}

Keywords: ternary cubic, non-homogeneous cubic, integer solutions.

2010 Mathematics subject classification: 11D25

\section{Notations Used}

$\mathrm{t}_{\mathrm{m}, \mathrm{n}}=$ Polygonal number of rank $\mathrm{n}$ with sides $\mathrm{m} .-\mathrm{p}_{\mathrm{n}}{ }^{\mathrm{m}}=$ Pyramidal number of rank $\mathrm{n}$ with size $\mathrm{m}$.

$\mathrm{G}_{\mathrm{n}}=$ Gnomonic number

\section{Introduction}

The theory of Diophantine equations offers a rich variety of fascinating problems. The non-homogeneous ternary cubic equation offers an unlimited field for research because of their variety

[1-2].For an extensive review of various problems one may refer [3-12]. This communication concerns with yet another interesting ternary cubic equation $x^{2}+y^{2}-x y=103 z^{2}$ for determining its infinitely many non-zero integral solutions. Also a few interesting properties among the solutions and special numbers are given.

\section{Method of Analysis}

The ternary cubic equation under consideration

$$
x^{2}+y^{2}-x y=103 z^{3}
$$

Introducing the linear transformations

$$
\begin{aligned}
& x=u+v, y=u-v, u \neq v \neq 0) \\
& \text { in (1), it simplifies to } u^{2}+3 v^{2}=103 z^{3}
\end{aligned}
$$

Taking $\mathrm{z}=\mathrm{z}(\mathrm{a}, \mathrm{b})=\mathrm{a}^{2}+3 \mathrm{~b}^{2}=(\mathrm{a}+\mathrm{i} \sqrt{3} \mathrm{~b})(\mathrm{a}-\mathrm{i} \sqrt{3} \mathrm{~b})$

where $a$ and $b$ non-zero distinct integers, different patterns of solutions of (1) are given below.

\subsection{Pattern: I}

$$
\text { Let } 103=(10+i \sqrt{3})(10-i \sqrt{3})
$$

Substituting (4) and (5) in (3) and employing the method of factorization, we have

$$
\begin{aligned}
& (u+i \sqrt{3} v)(u-i \sqrt{3} v)=(10+i \sqrt{3})(10-i \sqrt{3}) \\
& (a+i \sqrt{3} b)^{3}(a-i \sqrt{3} b)^{3}
\end{aligned}
$$

Equating the positive and negative factors, we get

$$
(u+i \sqrt{3} v)=(10+i \sqrt{3})(a+i \sqrt{3} b)^{3}
$$

$$
(u-i \sqrt{3} v)=(10-i \sqrt{3})(a-i \sqrt{3} b)
$$

Equating the real and imaginary parts in (6) or (7), we have $u=u(a, b)=10 a^{3}-60 a b^{2}-6 a^{2} b+9 b^{3}$

$\mathrm{v}=\mathrm{v}(\mathrm{a}, \mathrm{b})=\mathrm{a}^{3}-6 \mathrm{a} \mathrm{b}^{2}+20 \mathrm{a}^{2} \mathrm{~b}-30 \mathrm{~b}^{3}$ In view of (2), the values of $x, y$ are given by

$$
\begin{aligned}
& x=x(\mathrm{a}, \mathrm{b})=11 \mathrm{a}^{3}-66 \mathrm{ab}^{2}+14 \mathrm{a}^{2} \mathrm{~b}-21 \mathrm{~b}^{3} \\
& y=y(\mathrm{a}, \mathrm{b})=9 \mathrm{a}^{3}-54 \mathrm{ab}^{2}-26 \mathrm{a}^{2} \mathrm{~b}+39 \mathrm{~b}^{3}
\end{aligned}
$$

Thus (4), (8) and (9) represent the non-zero distinct integral solutions of (1) in two parameters.

\section{Properties:}

1. $\mathrm{y}(\mathrm{a}, \mathrm{a})-\mathrm{x}(\mathrm{a}, \mathrm{a})-232 \mathrm{P}_{\mathrm{a}}^{5}+116 \mathrm{t}_{4, \mathrm{a}}=0$

2. $\mathrm{z}(\mathrm{a}, \mathrm{a})-4 \mathrm{t}_{4, \mathrm{a}}=0$

3. $\mathrm{x}(\mathrm{a}, 1)-22 \mathrm{P}_{\mathrm{a}}^{5}+3 \mathrm{t}_{4, \mathrm{a}}+\mathrm{G}_{33 \mathrm{a}}+21=0$

4. $\mathrm{x}(1, \mathrm{~b})+42 \mathrm{P}_{\mathrm{b}}{ }^{5}+45 \mathrm{t}_{4, \mathrm{~b}}-\mathrm{G}_{7 \mathrm{~b}} \equiv 0(\operatorname{Mod} 11)$

5. $\mathrm{y}(1, \mathrm{~b})-78 \mathrm{P}_{\mathrm{b}}^{5}+93 \mathrm{t}_{4, \mathrm{~b}}+\mathrm{G}_{13 \mathrm{~b}} \equiv 0(\operatorname{Mod} 3)$.

6. $y(2 a, 2 a)+532 P_{b}{ }^{5}-266 t_{4, a}=0$.

7. $\mathrm{x}(3 \mathrm{a}, 4 \mathrm{a})+7430 \mathrm{P}_{\mathrm{b}}^{5}-3715 \mathrm{t}_{4, \mathrm{a}}=0$

8. $x(b,-1)-22 P_{b}^{5}+25 t_{4, b}+G 33 b \equiv 0(\operatorname{Mod} 11)$

\subsection{Pattern: II}

$$
\text { Let } 103=\frac{(20+i 2 \sqrt{3})(20-i 2 \sqrt{3})}{4}
$$

Substituting (4) and (10) in (3) and employing the Method of factorization, we have $(u+i \sqrt{3} v)(u-i \sqrt{3} v)=\frac{1}{4}[(20+i 2$

$\left.\sqrt{3})(20-i 2 \sqrt{3})(a+i \sqrt{3} b)^{3}(a-i \sqrt{3} b)^{3}\right]$

Equating the positive and negative factors, we get

$$
\begin{aligned}
& (\mathrm{u}+\mathrm{i} \sqrt{3} \mathrm{v})=\frac{1}{2}\left[(20+\mathrm{i} 2 \sqrt{3})(a+i \sqrt{3} b)^{3}\right] \\
& (\mathrm{u}-\mathrm{i} \sqrt{3} \mathrm{v})=\frac{1}{2}\left[(20-\mathrm{i} 2 \sqrt{3})(\mathrm{a}-\mathrm{i} \sqrt{3} \mathrm{~b})^{3}\right]
\end{aligned}
$$

Equating the real and imaginary parts in (11) or (12), we have

\section{Volume 5 Issue 3, March 2016}




\section{International Journal of Science and Research (IJSR) \\ ISSN (Online): 2319-7064}

Index Copernicus Value (2013): 6.14 | Impact Factor (2014): 5.611

$\mathrm{u}=\mathrm{u}(\mathrm{a}, \mathrm{b})=\frac{1}{2}\left[20 \mathrm{a}^{3}-180 a b^{2}-18 \mathrm{a}^{2} \mathrm{~b}+18 \mathrm{~b}^{3}\right]$

$\mathrm{v}=\mathrm{v}(\mathrm{a}, \mathrm{b})=\frac{1}{2}\left[2 \mathrm{a}^{3}-18 \mathrm{ab} \mathrm{b}^{2}+60 \mathrm{a}^{2} \mathrm{~b}-60 \mathrm{~b}^{3}\right]$

In view of (2), the values of $x, y$ are given by

$$
\begin{gathered}
x=x(a, b)=11 a^{3}-99 a b^{2}-21 a^{2} b+21 b^{3} \\
y=y(a, b)=9 a^{3}-81 a b^{2}-39 a^{2} b+39 b^{3}
\end{gathered}
$$

Thus (4), (13) and (14) represents the non-zero distinct integral solution of (1) in two parameters.

\section{Properties:-}

1. $y(a, a)-x(a, a)+32 P_{a}{ }^{5}-16 t_{4, a}=0$

2. $9 \mathrm{x}(\mathrm{b}, \mathrm{b})-11 \mathrm{y}(\mathrm{b}, \mathrm{b})=0$

3. $\mathrm{x}(\mathrm{a}, 1)-22 \mathrm{P}_{\mathrm{a}}^{5}+99 \mathrm{P}_{\mathrm{a}}-109 \mathrm{t}_{4, \mathrm{a}}+21=0$

4. $\mathrm{y}(1, \mathrm{~b})-78 \mathrm{P}_{\mathrm{b}}^{5}+81 \mathrm{t}_{4, \mathrm{~b}}-39 \mathrm{P}_{\mathrm{b}} \equiv 0(\operatorname{Mod} 3)$

5. $\mathrm{z}(\mathrm{a}, 2 \mathrm{a})-\mathrm{t}_{28, \mathrm{a}}-\mathrm{G}_{6 \mathrm{n}}-1=0$

6. $\mathrm{y}(-1, \mathrm{~b})-78 \mathrm{P}_{\mathrm{b}}^{5}+39 \mathrm{P}_{\mathrm{b}}+81 \mathrm{t}_{4, \mathrm{~b}} \equiv 0(\operatorname{Mod} 3)$

7. $x(2 \mathrm{a}, 1)-176 \mathrm{P}_{\mathrm{b}}^{5}+4 \mathrm{t}_{4, \mathrm{a}}+\mathrm{G}_{99 \mathrm{~b}} \equiv 0(\operatorname{Mod} 2)$

8. $\mathrm{y}(4, \mathrm{~b})-78 \mathrm{P}_{\mathrm{b}}^{5}+363 \mathrm{t}_{4, \mathrm{~b}}+\mathrm{G}_{312, \mathrm{~b}}-577=0$.

\subsection{Pattern III}

$$
\begin{aligned}
& \text { Write }(3) \text { as } \mathrm{u}^{2}+3 \mathrm{v}^{2}=103 \mathrm{z}^{3} * 1 \\
& \text { Write } 1 \text { as } 1=\frac{(1+i \sqrt{3})(1-i \sqrt{3})}{4}
\end{aligned}
$$

Substituting (4), (5) and (16) in (15) and employing the method of factorization, we have

$$
\begin{aligned}
& (u+i \sqrt{3} v)(u-i \sqrt{3} v)=\frac{1}{4}[(1+i \sqrt{3})(1-i \sqrt{3}) \\
& \left.(10+i \sqrt{3})(10-i \sqrt{3})(a+i \sqrt{3} b)^{3}(a-i \sqrt{3} b)^{3}\right]
\end{aligned}
$$

Equating the positive and negative factors, we get

$$
\begin{aligned}
& (u+i \sqrt{3} v)=\frac{1}{2}\left[(1+i \sqrt{3})(10+i \sqrt{3})(a+i \sqrt{3} b)^{3}\right](17) \\
& (u-i \sqrt{3} v)=\frac{1}{2}\left[(1-i \sqrt{3})(10-i \sqrt{3})(a-i \sqrt{3} b)^{3}\right]
\end{aligned}
$$

Equating the real and imaginary parts in (17) or (18), we have

$$
\mathrm{u}=\mathrm{u}(\mathrm{a}, \mathrm{b})=\frac{1}{2}\left[7 \mathrm{a}^{3}-63 a b^{2}-99 \mathrm{a}^{2} \mathrm{~b}+99 \mathrm{~b}^{3}\right]
$$$$
\mathrm{v}=\mathrm{v}(\mathrm{a}, \mathrm{b})=\frac{1}{2}\left[11 \mathrm{a}^{3}-99 \mathrm{ab} \mathrm{b}^{2}+21 \mathrm{a}^{2} \mathrm{~b}-21 \mathrm{~b}^{3}\right]
$$

In view of (2), the values of $x, y$ are given by

$$
\begin{gathered}
x=x(a, b)=9 a^{3}-81 a b^{2}-39 a^{2} b+39 b^{3} \\
y=y(a, b)=-2 a b^{3}+18 a b^{2}-60 a^{2} b+60 b^{3}
\end{gathered}
$$

\section{Properties:-}

1. $\mathrm{y}(\mathrm{a}, \mathrm{a})-\mathrm{x}(\mathrm{a}, \mathrm{a})-176 \mathrm{P}_{\mathrm{a}}{ }^{5}+88 \mathrm{t}_{4, \mathrm{a}}=0$

2. $\mathrm{x}(1, \mathrm{~b})+78 \mathrm{P}_{\mathrm{a}}^{5}+39 \mathrm{P}_{\mathrm{b}}+81 \mathrm{t}_{4,9} \equiv 0(\operatorname{Mod} 3)$

3. $x(b, b)+9 y(b, b)=0$

4. $\mathrm{z}(\mathrm{b}, \mathrm{b})-4 \mathrm{t}_{4, \mathrm{~b}}=0$

5. y $(1, \mathrm{~b})-60 \mathrm{P}_{\mathrm{b}}-138 \mathrm{t}_{4, \mathrm{~b}} \equiv 0(\operatorname{Mod} 2)$

6. $x(b, 4)-18 P_{b}^{5}+165 t_{4, b}+G_{648, b}-2497=0$

7. $9 x(b, b)-2 y(b, b)+1232 \mathrm{P}_{\mathrm{b}}^{5}-616 \mathrm{t}_{4, \mathrm{~b}}=0$

8. $\mathrm{z}(4 \mathrm{a}, 3 \mathrm{a})-43 \mathrm{t}_{4, \mathrm{a}}=0$.

\subsection{Pattern IV}

Instead of (16),
Write 1 as $\frac{(1+i 4 \sqrt{3})(1-i 4 \sqrt{3})}{49}$

Substituting (4), (10) and (21) in (15) and employing the method of factorization, we have

$$
(\mathrm{u}+\mathrm{i} \sqrt{3} \mathrm{v})(\mathrm{u}-\mathrm{i} \sqrt{3} \mathrm{v})=\frac{1}{4 \times 49}[(1+\mathrm{i} 4 \sqrt{3})(1-\sqrt{3})
$$

$$
\left.(20-i 2 \sqrt{3})(20+i 2 \sqrt{3})(a+i \sqrt{3} b)^{3}(a-i \sqrt{3} b)^{3}\right]
$$

Equating the positive and negative factors, we get

$$
\begin{aligned}
& (u+i \sqrt{3} v)=\frac{1}{14}\left[(1+i 4 \sqrt{3})(20+i 2 \sqrt{3})(a+i \sqrt{3} b)^{3}\right] \\
& (u-i \sqrt{3} v)=\frac{1}{14}\left[(1-i 4 \sqrt{3})(20-i 2 \sqrt{3})(a-i \sqrt{3} b)^{3}\right]
\end{aligned}
$$

Equating the real and imaginary parts in (22) or (23), we have

$$
\begin{aligned}
& \mathrm{u}=\mathrm{u}(\mathrm{a}, \mathrm{b})=\frac{1}{14}\left[-4 \mathrm{a}^{3}-36 a b^{2}-738 \mathrm{a}^{2} \mathrm{~b}+738 \mathrm{~b}^{3}\right] \\
& \mathrm{v}=\mathrm{v}(\mathrm{a}, \mathrm{b})=\frac{1}{14}\left[82 \mathrm{a}^{3}-738 \mathrm{ab}-12 \mathrm{a}^{2} \mathrm{~b}+12 \mathrm{~b}^{3}\right]
\end{aligned}
$$

In view of (2), the values of $x, y$ are given by

$$
\begin{aligned}
& x=x(a, b)=\frac{1}{7}\left[39 a^{3}-387 a b^{2}-375 a^{2} b+375 b^{3}\right] \\
& y=y(a, b)=\frac{1}{7}\left[-48 a^{3}+351 a b^{2}-363 a^{2} b+363 b^{3}\right]
\end{aligned}
$$

As our interest in on finding integer solutions, replacing a by $7 \mathrm{a}$ and $\mathrm{b}$ by $7 \mathrm{~b}$ in (4), (24) and (25), the corresponding integer solutions of (1) in two parameters are given by $\mathrm{x}=\mathrm{x}(\mathrm{a}, \mathrm{b})=-1911 \mathrm{a}^{3}+18963 \mathrm{ab}^{2}-18375 \mathrm{a}^{2} \mathrm{~b}+18375 \mathrm{~b}^{3}$ $y=y(a, b)=-2352 a^{3}+17199 a b^{2}-17787 a^{2} b+17787 b^{3}$ $\mathrm{z}=\mathrm{z}(\mathrm{a}, \mathrm{b})=49 \mathrm{a}^{2}+147 \mathrm{~b}^{2}$

\section{Properties:}

1. $x(a, a)-y(a, a)+63798 P_{a}^{5}-31899 t_{4, a}=0$

2. $\mathrm{z}(\mathrm{a}, \mathrm{a})-\mathrm{t}_{394, \mathrm{a}}-\mathrm{G}_{97 \mathrm{a}}-\mathrm{P}_{\mathrm{a}}-1+\mathrm{t}_{4, \mathrm{a}}=0$

3. $\mathrm{x}(1, \mathrm{~b})-36750 \mathrm{P}_{\mathrm{b}}^{5}+18375 \mathrm{P}_{\mathrm{b}}+18963 \mathrm{t}_{4, \mathrm{~b}}+1911=0$

4. $\mathrm{y}(\mathrm{a}, 1)+4704 \mathrm{P}_{\mathrm{a}}{ }^{5}+17199 \mathrm{P}_{\mathrm{a}}+32634 \mathrm{t}_{4, \mathrm{a}}-17787=0$

5. $\mathrm{z}(\mathrm{a}, 1)-49 \mathrm{t}_{4, \mathrm{a}} \equiv 0(\operatorname{Mod} 7)$

6. $\mathrm{x}(\mathrm{a},-\mathrm{a})+34104 \mathrm{P}_{\mathrm{b}}^{5}-17052 \mathrm{t}_{4, \mathrm{a}}=0$

7. $y(a,-a)-29694 \mathrm{P}_{\mathrm{b}}^{5}+14847 \mathrm{t}_{4, \mathrm{a}}=0$

$8 . z(a,-a)-196 t_{4, a}=0$.

\section{Conclusion}

In this paper, we have illustrated different methods of obtaining infinitely Many non-zero distinct integer solutions to the cubic equations $x^{2}+y^{2}-x y=103 z^{2}$ are rich in variety, one may search for non -zero distinct integer solutions to the other choices of cubic equations with three or more variables along with their corresponding properties.

\section{References}

[1] Dickson, L.E., History of theory of numbers, Vol.11, Chelsea publishing company, New -York (1952)

[2] Mordell, L.J., Diophantine equation, Academic press, London (1969) Journal of Science and Research, Vol (3), Issue 12, 20-22 (December -14)

[3] Jayakumar. P, Sangeetha, K "Lattice points on the cone $\mathrm{x}^{2}+9 \mathrm{y}^{2}=50 \mathrm{z}^{2}$ " International Journal of Science and Research, Vol (3), Issue 12, 20-22 (December -2014) 
[4] Jayakumar P, Kanaga Dhurga, C," On Quadratic Diopphantine equation $\mathrm{x}^{2}+16 \mathrm{y}^{2}=20 \mathrm{z}^{2}$ " Galois $\mathrm{J}$. Maths, 1(1) (2014), 17-23.

[5] Jayakumar. P, Kanaga Dhurga. C, "Lattice points on the cone $x^{2}+9 y^{2}=50 z^{2}$ " Diophantus J. Math, 3(2) (2014), $61-71$

[6] Jayakumar. P, Prabha. S "On Ternary Quadratic Diophantine equation $\mathrm{x}^{2}+15 \mathrm{y}^{2}=14 \mathrm{z}^{2}$ " Archimedes J. Math., 4(3) (2014), 159-164.

[7] Jayakumar, P, Meena, J "Integral solutions of the Ternary Quadratic Diophantine equation $x^{2}+7 y^{2}=16 z^{2}$ International Journal of Science and Technology, Vol.4, Issue 4, 1-4, Dec 2014.

[8] Jayakumar. P, Shankarakalidoss, G "Lattice point on Homogenous cone $\mathrm{x}^{2}+9 \mathrm{y}^{2}=50 \mathrm{z}^{2}$, International journal of Science and Research, Vol (4), Issue 1, 2053-2055, January -2015 .

[9] Jayakumar. P, Shankarakalidoss. G "Integral points on the Homogenous cone $x^{2}+y^{2}=10 z^{2}$ International Journal for Scienctific Research and Development, Vol (2), Issue 11, 234-235, January -2015

[10] Jayakumar.P, Prapha.S "Integral points on the cone $x^{2}$ $+25 \mathrm{y}^{2}=17 \mathrm{z}^{2, '}$ International Journal of Science and Research Vol(4), Issue 1, 2050-2052, January-2015.

[11] Jayakumar.P, Prabha. S, "Lattice points on the cone $x^{2}+$ $9 \mathrm{y}^{2}=26 \mathrm{z}^{2}$ "International Journal of Science and Research Vol (4), Issue 1, 2050-2052, January 2015

[12] Jayakumar. P, Sangeetha. K, "Integral solution of the Homogeneous Biquadratic Diophantine equation with six unknowns: $\left(\mathrm{x}^{3}-\mathrm{y}^{3}\right) \mathrm{z}=\left(\mathrm{W}^{2}-\mathrm{P}^{2}\right) \mathrm{R}^{4}$ "International Journal of Science and Research, Vol(3), Issue 12, 1021-1023 (December-2014)

\section{Author Profile}

P. Jayakumar received the B. Sc, M.Sc degrees in Mathematics from Madras University in 1980 and 1982 and the M. Phil, Ph.D degrees in Mathematics from Bharathidasan University, Thiruchirappalli in 1988 and 2010. Who is now working as Professor of Mathematics, Periyar Maniammai University,Vallam, Thanajvur-613403, Tamil Nadu, India

J. Meena received the B. Sc, M.Sc degrees in Mathematics from Bharathidasan University, Thiruchirappalli in 2008 and in 2010 and the M. Phil., degree in Mathematics from Prist University, Thanjavur in 2013. Who is now working as Assistant Professor of Mathematics, A.V.V.M Sri Pushpam College Poondi (Autonomous), Thanjavur (District) - 613 503, Tamil Nadu, India. 